Brief Report

\title{
Assessment of In Vitro Cefiderocol Susceptibility and Comparators against an Epidemiologically Diverse Collection of Acinetobacter baumannii Clinical Isolates
}

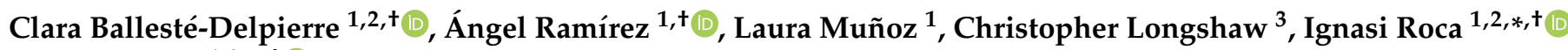 \\ and Jordi Vila $1,2, *,+$ (iD
}

check for

updates

Citation: Ballesté-Delpierre, C.;

Ramírez, Á.; Muñoz, L.; Longshaw,

C.; Roca, I.; Vila, J. Assessment of In Vitro Cefiderocol Susceptibility and Comparators against an Epidemiologically Diverse Collection of Acinetobacter baumannii Clinical Isolates. Antibiotics 2022, 11, 187. https://doi.org/10.3390/ antibiotics 11020187

Academic Editor: Marcello Covino

Received: 31 December 2021

Accepted: 29 January 2022

Published: 31 January 2022

Publisher's Note: MDPI stays neutral with regard to jurisdictional claims in published maps and institutional affiliations.

Copyright: (C) 2022 by the authors. Licensee MDPI, Basel, Switzerland. This article is an open access article distributed under the terms and conditions of the Creative Commons Attribution (CC BY) license (https:// creativecommons.org/licenses/by/ $4.0 /)$.
1 Department of Clinical Microbiology, ISGlobal, Hospital Clínic-Universitat de Barcelona, Rosselló 149-153, 08036 Barcelona, Spain; clara.balleste@isglobal.org (C.B.-D.); aramirma47@alumnes.ub.edu (Á.R.); laura.munoz@isglobal.org (L.M.)

2 CIBER Enfermedades Infecciosas, 28029 Madrid, Spain

3 Infectious Diseases, Shionogi B.V., London WC2B 6UF, UK; christopher.longshaw@shionogi.eu

* Correspondence: Ignasi.roca@isglobal.org (I.R.); jvila@ub.edu (J.V.); Tel.: +34-93-2275400 (I.R. \& J.V.)

+ These authors contributed equally to this work.

\begin{abstract}
Cefiderocol is a catechol-substituted siderophore cephalosporin combining rapid penetration into the periplasmic space with increased stability against $\beta$-lactamases. This study provides additional data on the in vitro antimicrobial activity of cefiderocol and commercially available comparators against an epidemiologically diverse collection of Acinetobacter baumannii clinical isolates. Antimicrobial susceptibility was tested using pre-prepared frozen 96-well microtiter plates containing twofold serial dilutions of: cefepime, ceftazidime/avibactam, imipenem/relebactam, ampicillin/sulbactam, meropenem, meropenem/vaborbactam, ciprofloxacin, minocycline, tigecycline, trimethoprim/sulfamethoxazole and colistin using the standard broth microdilution procedure in cation-adjusted Mueller-Hinton broth (CAMHB). For cefiderocol, iron-depleted CAMHB was used. A collection of 113 clinical strains of A. baumannii isolated from Argentina, Azerbaijan, Croatia, Greece, Italy, Morocco, Mozambique, Peru and Spain were included. The most active antimicrobial agents against our collection were colistin and cefiderocol, with $12.38 \%$ and $21.23 \%$ of non-susceptibility, respectively. A high proportion of multidrug-resistant (76.77\%) and carbapenemresistant (75.28\%) A. baumannii isolates remained susceptible to cefiderocol, which was clearly superior to novel $\beta$-lactam $/ \beta$-lactamase inhibitor combinations. Cefiderocol-resistance was higher among carbapenem-resistant isolates and isolates belonging to ST2, but could not be associated with any particular resistance mechanism or clonal lineage. Our data suggest that cefiderocol is a good alternative to treat infections caused by MDR A. baumanni, including carbapenem-resistant strains.
\end{abstract}

Keywords: Acinetobacter baumannii; antibiotic resistance; carbapenem; MDR; trojan horse; cefiderocol; siderophore; epidemiology

\section{Introduction}

The emergence of bacteria resistant to currently available antibiotics is steadily increasing but the development of new therapies to combat infections caused by these microorganisms are not following at an appropriate pace. In the clinical setting infections caused by multidrug-resistant (MDR), Gram-negative bacteria are steadily increasing and of major global concern, particularly those caused by clinical isolates of Enterobacterales and non-fermentative Gram-negative bacilli, mainly Pseudomonas aeruginosa and Acinetobacter baumannii. Among the antibiotics towards which these bacteria show a resistant phenotype, carbapenem resistance deserves special attention as carbapenems are used as a therapy of last resort [1]. 
In 2017, the World Health Organization published a global priority report of antibioticresistant microorganisms to guide research and development of new antibacterial agents. Carbapenem-resistant A. baumannii is on top of this list [2]. According to the 2018 EARSNet publication, more than half of the Acinetobacter spp. isolates reported by EU/EEA countries were resistant to at least one of the three antimicrobial groups: fluoroquinolones, aminoglycosides and carbapenems (representing $36.2 \%$ for fluoroquinolone-resistance and $31.9 \%$ in the other two remaining groups) [3]. Data from the Centre for Disease Control (CDC) indicate that annually, 8500 infections are caused by carbapenem-resistant Acinetobacter in the United States [4]. Current therapeutic options for the treatment of carbapenem-resistant $A$. baumannii infections are limited and suboptimal, being restricted to available antibiotics including colistin, tigecycline, minocycline, amikacin and sulbactam. These antimicrobials are administered either alone or in combination, despite the occurrence of pharmacokinetic issues and high toxicity in some cases, as it is the case for colistin and amikacin [5].

Several strategies to discover new antibacterial agents are currently being explored, such as the chemical modification of existing antimicrobial agents [6]. Among these, cefiderocol (Shionogi Inc.) is likely one of the most promising molecules recently released [7]. Cefiderocol is a novel catechol-substituted siderophore cephalosporin that combines rapid penetration into the periplasmic space via iron transport and increased stability to enzymatic hydrolysis by all Ambler classes of $\beta$-lactamases. In vitro, preclinical and clinical studies have shown expanded activity against MDR bacteria compared to commercialized antibiotics [8]. In November 2019, cefiderocol was approved for the treatment of adults with complicated urinary tract infections by the Food and Drug Administration (FDA) [9] and, in May 2020, for the treatment of complicated Gram-negative infections by the European Medicines Agency [10].

In the current study, a collection of 113 A. baumannii clinical isolates including carbapenemresistant and MDR isolates from nine countries across the globe was tested against cefiderocol and relevant comparative antibacterial agents following the Clinical and Laboratory Standards Institute (CLSI) broth microdilution method. These isolates were characterized from an epidemiological point of view as well as in terms of carbapenem-resistance and the carriage of carbapenem-resistant genes.

\section{Results and Discussion}

\subsection{Susceptibility Profiles against Cefiderocol and Comparators}

The present study comprised a collection of 113 bacterial isolates of A. baumannii recovered from several countries all over the world including both carbapenem-resistant and carbapenem-susceptible isolates. Upon testing the antimicrobial susceptibility to a selected panel of antibacterial agents, the lowest MIC $_{50}$ values reported among all 113 isolates tested corresponded to cefiderocol and colistin $\left(\mathrm{MIC}_{50}=0.5 \mu \mathrm{g} / \mathrm{mL}\right)$, closely followed by tigecycline and minocycline ( $\mathrm{MIC}_{50}=2$ and $4 \mu \mathrm{g} / \mathrm{mL}$, respectively). The $\mathrm{MIC}_{90}$ values, however, showed a slightly different pattern. Tigecycline showed the lowest $\mathrm{MIC}_{90}$ value $\left(\mathrm{MIC}_{90}=4 \mu \mathrm{g} / \mathrm{mL}\right.$ ) followed by colistin $\left(\mathrm{MIC}_{90}=8 \mu \mathrm{g} / \mathrm{mL}\right.$ ). Cefiderocol, on the other hand, presented a $\mathrm{MIC}_{90}$ of $>64 \mu \mathrm{g} / \mathrm{mL}$, only comparable to that of ampicillin/sulbactam (Table 1). While these values were above the range detected in previously published studies, Isler et al. have shown important differences in the reported $\mathrm{MIC}_{50}-\mathrm{MIC}_{90}$ values depending on the collection of strains being tested [5]. 
Table 1. Minimum inhibitory concentration (MIC, $\mu \mathrm{g} / \mathrm{mL}$ ) for cefiderocol and comparators against a collection of 113 A. baumannii clinical isolates. Clinical and Laboratory Standards Institute (CLSI) breakpoints were applied to determine categories of susceptible, intermediate and resistant.

\begin{tabular}{|c|c|c|c|c|c|c|}
\hline \multirow{2}{*}{ Antimicrobial Agents } & $\mathrm{MIC}_{50}$ & $\mathrm{MIC}_{90}$ & Range & $\mathbf{S}$ & $\mathbf{I}$ & $\mathbf{R}$ \\
\hline & \multicolumn{3}{|c|}{$\mu \mathrm{g} / \mathrm{mL}$} & \multicolumn{3}{|c|}{ n (\%) } \\
\hline Cefiderocol & 0.5 & $>64$ & $\leq 0.03$ to $>64$ & $90(79.60)$ & $1(0.88)$ & $23(20.35)$ \\
\hline Cefepime & $>16$ & $>16$ & $\leq 0.125$ to $>16$ & 15 (13.27) & $4(3.54)$ & $94(83.18)$ \\
\hline Ceftazidime/avibactam ${ }^{\mathrm{a}}$ & $>16$ & $>16$ & $\leq 0.125$ to $>16$ & NA & NA & NA \\
\hline Meropenem & $>16$ & $>16$ & 0.125 to $>16$ & $22(19.47)$ & $2(1.77)$ & $89(78.76)$ \\
\hline Meropenem/vaborbactam ${ }^{a}$ & $>16$ & $>16$ & 0.125 to $>16$ & NA & NA & NA \\
\hline Imipenem/relebactam $^{\mathrm{a}}$ & $>16$ & $>16$ & 0.125 to $>16$ & NA & NA & NA \\
\hline Ampicillin-sulbactam & 32 & 64 & $\leq 2$ to $>64$ & $24(21.24)$ & $20(17.70)$ & $69(61.06)$ \\
\hline Ciprofloxacin & $>8$ & $>8$ & $\leq 0.125$ to $>8$ & $12(10.62)$ & $0(0.00)$ & $101(89.38)$ \\
\hline Minocycline & 4 & $>8$ & $\leq 0.25$ to $>8$ & $65(57.52)$ & $21(18.58)$ & $27(23.89)$ \\
\hline Tigecycline $^{\mathrm{a}}$ & 2 & 4 & $\leq 0.125$ to $>4$ & NA & NA & NA \\
\hline $\begin{array}{l}\text { Trimethoprim- } \\
\text { sulfamethoxazole }\end{array}$ & $>8$ & $>8$ & $\leq 0.25$ to $>8$ & $19(16.81)$ & NA & $94(83.18)$ \\
\hline Colistin ${ }^{b}$ & 0.5 & 8 & $\leq 0.25$ to $>8$ & NA & $99(87.61)$ & $14(12.39)$ \\
\hline
\end{tabular}

a No breakpoints available at CLSI; ${ }^{\mathrm{b}}$ The CLSI update released in April 2021 removed the susceptible breakpoint for colistin. Only intermediate $(\leq 2 \mu \mathrm{g} / \mathrm{mL})$ and resistant $(\geq 4 \mu \mathrm{g} / \mathrm{mL})$ breakpoints for colistin are now available; $\mathrm{MIC}_{50}$ indicates the MIC value at which $50 \%$ of isolates tested are inhibited; $\mathrm{MIC}_{90}$ indicates the MIC value at which $90 \%$ of isolates tested are inhibited. S, susceptible; I, intermediate; R, resistant. NA, not applicable.

Nevertheless, the most active antimicrobial agents in our collection of isolates were both colistin and cefiderocol, showing only $12.38 \%$ of resistant isolates and $21.23 \%$ of non-susceptible isolates, respectively. Minocycline also showed good activity overall with $23.89 \%$ of resistant isolates. It is worth mentioning that more than $80 \%$ of the isolates were resistant to all of the remaining antimicrobial agents and only a handful of isolates showed an overall susceptible profile (Table 2). As expected, a very high proportion of isolates were non-susceptible to cephalosporins, regardless of the carriage of any carbapenemase or meropenem susceptibility, with $86.76 \%(98 / 113)$ of the isolates being non-susceptible to cefepime and $94.69 \%(107 / 113)$ showing MIC values of ceftazidime/avibactam $\geq 16 \mu \mathrm{g} / \mathrm{mL}$.

As much as $87.61 \%(99 / 113)$ of the $A$. baumannii isolates included in this study were non-susceptible to at least one antimicrobial agent from three classes tested (13 of them being already resistant to colistin) and, hence, were considered as MDR [11]. Among MDR isolates, $76.77 \%$ (76/99) were susceptible to cefiderocol with MIC values of $\leq 8 \mu \mathrm{g} / \mathrm{mL}$, but all isolates that were resistant to cefiderocol were also MDR. In addition, $72.72 \%$ (72/99) and $92.92 \%$ (92/99) of MDR isolates were also susceptible to minocycline and tigecycline, respectively, both of them being considered as valuable therapeutic alternatives for the treatment of infections caused by MDR A. baumannii [12]. Among minocyclineresistant $A$. baumannii, three isolates were resistant to cefiderocol but remained susceptible to tigecycline and colistin. The few tigecycline-resistant isolates were all susceptible to cefiderocol and only two of them showed resistance to colistin (Table 2). 
Table 2. Antibiotic susceptibility and molecular characterization of A. baumannii bacterial isolates used in this study.

\begin{tabular}{|c|c|c|c|c|c|c|c|c|c|c|c|c|c|c|c|c|c|}
\hline \multirow{2}{*}{ ID } & \multirow{2}{*}{ Country } & CFDC & CST & MIN & CIP & SXT & TGC & SAM & FEP & CZA & MEM & MEV & IPR & \multirow{2}{*}{ aCarb } & \multirow{2}{*}{ MDR } & \multirow{2}{*}{ ST } & \multirow{2}{*}{$\mathrm{CC}$} \\
\hline & & \multicolumn{12}{|c|}{$\mu \mathrm{g} / \mathrm{mL}$} & & & & \\
\hline SHG-44 & Argentina & 0.125 & 2 & 1 & $>8$ & $>8$ & 1 & 32 & $>16$ & $>16$ & $>16$ & $>16$ & $>16$ & OXA-23 & MDR & 1 & $\mathrm{CC} 1$ \\
\hline SHG-43 & Argentina & 0.25 & 1 & 8 & $>8$ & $>8$ & 2 & 32 & $>16$ & $>16$ & $>16$ & $>16$ & $>16$ & OXA-23 & MDR & 2 & CC2 \\
\hline SHG-45 & Argentina & 0.5 & 0.5 & 8 & $>8$ & $>8$ & 2 & 64 & $>16$ & 16 & $>16$ & $>16$ & $>16$ & OXA-23 & MDR & 2 & CC2 \\
\hline SHG-46 & Argentina & 0.125 & 0.25 & 0.25 & 0.125 & 0.25 & 0.125 & 2 & 2 & 8 & 0.5 & 0.5 & 0.5 & ND & - & 404 & CC3 \\
\hline SHG-94 & Azerbaijan & 1 & 0.5 & 1 & $>8$ & $>8$ & 0.5 & 64 & $>16$ & 16 & 8 & 2 & 1 & ND & MDR & 19 & CC1 \\
\hline SHG-112 & Azerbaijan & 0.25 & 0.25 & 0.5 & $>8$ & $>8$ & 0.5 & 64 & $>16$ & $>16$ & $>16$ & $>16$ & $>16$ & OXA-40 & MDR & 78 & CC1017 \\
\hline SHG-108 & Azerbaijan & 0.5 & 0.25 & 0.25 & $>8$ & 0.25 & 0.25 & 64 & $>16$ & $>16$ & $>16$ & $>16$ & $>16$ & OXA-40 & MDR & 78 & CC1017 \\
\hline SHG-110 & Azerbaijan & 0.5 & 0.25 & 0.25 & $>8$ & 0.25 & 0.125 & 8 & 4 & 16 & $>16$ & $>16$ & $>16$ & OXA-40 & MDR & 1077 & CC1017 \\
\hline SHG-109 & Azerbaijan & 1 & 0.5 & 4 & $>8$ & $>8$ & 1 & 8 & $>16$ & $>16$ & $>16$ & $>16$ & $>16$ & OXA-40 & MDR & 1077 & CC1017 \\
\hline SHG-97 & Azerbaijan & 0.25 & 0.25 & 8 & $>8$ & $>8$ & 2 & 32 & $>16$ & $>16$ & 1 & 0.5 & 0.5 & ND & MDR & 2 & $\mathrm{CC} 2$ \\
\hline SHG-104 & Azerbaijan & 0.06 & 0.5 & 0.25 & 0.25 & 0.25 & 0.125 & 2 & 2 & 16 & 0.25 & 0.25 & 0.25 & ND & - & 1422 & CC3 \\
\hline SHG-105 & Azerbaijan & 1 & 4 & 0.25 & $>8$ & $>8$ & 0.5 & 8 & $>16$ & $>16$ & 0.5 & 0.5 & 0.5 & ND & MDR & 1422 & CC3 \\
\hline SHG-106 & Azerbaijan & 0.25 & 0.5 & 4 & $>8$ & $>8$ & 1 & 16 & $>16$ & $>16$ & $>16$ & $>16$ & $>16$ & OXA-23 & MDR & 625 & CC462 \\
\hline SHG-100 & Azerbaijan & 0.06 & 0.5 & 0.25 & 0.25 & 0.25 & 0.125 & 2 & 2 & 16 & 0.25 & 0.25 & 0.25 & ND & - & 578 & CC806 \\
\hline SHG-47 & Croatia & 0.25 & 2 & 8 & $>8$ & 1 & 2 & 16 & $>16$ & $>16$ & $>16$ & $>16$ & $>16$ & OXA-72 & MDR & 2 & CC2 \\
\hline SHG-58 & Greece & 0.5 & 0.5 & 1 & $>8$ & $>8$ & 2 & 32 & $>16$ & $>16$ & $>16$ & $>16$ & $>16$ & OXA-23 & MDR & 1 & CC1 \\
\hline SHG-55 & Greece & 2 & 2 & 2 & $>8$ & $>8$ & 2 & 64 & $>16$ & 16 & $>16$ & $>16$ & $>16$ & OXA-23 & MDR & 1 & CC1 \\
\hline SHG-57 & Greece & 0.125 & $>8$ & $>8$ & $>8$ & $>8$ & $>4$ & $>64$ & $>16$ & $>16$ & $>16$ & $>16$ & $>16$ & OXA-23 & MDR & 2 & CC2 \\
\hline SHG-53 & Greece & 0.25 & 0.25 & $>8$ & $>8$ & 2 & 2 & 64 & $>16$ & $>16$ & $>16$ & $>16$ & $>16$ & OXA-23 & MDR & 2 & CC2 \\
\hline SHG-56 & Greece & 0.5 & $>8$ & $>8$ & $>8$ & $>8$ & 4 & 64 & $>16$ & $>16$ & $>16$ & $>16$ & $>16$ & OXA-23 & MDR & 2 & CC2 \\
\hline SHG-54 & Greece & 1 & $>8$ & 4 & $>8$ & $>8$ & $>4$ & 32 & $>16$ & $>16$ & $>16$ & $>16$ & $>16$ & OXA-23 & MDR & 2 & $\mathrm{CC} 2$ \\
\hline SHG-52 & Greece & 4 & 8 & 8 & $>8$ & $>8$ & 2 & 64 & $>16$ & $>16$ & $>16$ & $>16$ & $>16$ & OXA-23 & MDR & 2 & CC2 \\
\hline SHG-65 & Italy & 0.5 & $>8$ & $>8$ & $>8$ & $>8$ & 2 & 64 & $>16$ & $>16$ & $>16$ & $>16$ & $>16$ & OXA-23 & MDR & 2 & CC2 \\
\hline SHG-66 & Italy & 1 & 0.5 & $>8$ & $>8$ & $>8$ & 1 & 64 & $>16$ & $>16$ & $>16$ & $>16$ & $>16$ & OXA-23 & MDR & 2 & CC2 \\
\hline SHG-61 & Italy & 2 & 8 & 4 & $>8$ & $>8$ & 2 & 16 & $>16$ & $>16$ & $>16$ & $>16$ & $>16$ & OXA-23 & MDR & 2 & $\mathrm{CC} 2$ \\
\hline SHG-63 & Italy & 2 & 8 & 4 & $>8$ & $>8$ & 2 & 16 & $>16$ & $>16$ & $>16$ & $>16$ & $>16$ & OXA-23 & MDR & 2 & $\mathrm{CC} 2$ \\
\hline SHG-62 & Italy & 4 & 0.5 & $>8$ & $>8$ & $>8$ & 2 & 64 & $>16$ & $>16$ & $>16$ & $>16$ & $>16$ & OXA-23 & MDR & 2 & $\mathrm{CC} 2$ \\
\hline
\end{tabular}


Table 2. Cont.

\begin{tabular}{|c|c|c|c|c|c|c|c|c|c|c|c|c|c|c|c|c|c|}
\hline \multirow{2}{*}{ ID } & \multirow{2}{*}{ Country } & CFDC & CST & MIN & CIP & SXT & TGC & SAM & FEP & CZA & MEM & MEV & IPR & \multirow{2}{*}{ aCarb } & \multirow{2}{*}{ MDR } & \multirow{2}{*}{ ST } & \multirow{2}{*}{$\mathrm{CC}$} \\
\hline & & \multicolumn{12}{|c|}{$\mu \mathrm{g} / \mathrm{mL}$} & & & & \\
\hline SHG-60 & Italy & 4 & $>8$ & 4 & $>8$ & $>8$ & 4 & 16 & $>16$ & $>16$ & $>16$ & $>16$ & $>16$ & OXA-23 & MDR & 2 & $\mathrm{CC} 2$ \\
\hline SHG-11 & Morocco & 1 & 0.25 & $>8$ & $>8$ & $>8$ & 1 & 64 & $>16$ & $>16$ & $>16$ & $>16$ & $>16$ & OXA-23/NDM & MDR & 315 & $\mathrm{CC} 1$ \\
\hline SHG-1 & Morocco & 1 & 0.5 & $>8$ & $>8$ & $>8$ & 2 & 64 & $>16$ & $>16$ & $>16$ & $>16$ & $>16$ & OXA-23 & MDR & 315 & $\mathrm{CC} 1$ \\
\hline SHG-10 & Morocco & 4 & 0.25 & 4 & $>8$ & $>8$ & 0.5 & 64 & $>16$ & $>16$ & $>16$ & $>16$ & $>16$ & OXA-23/NDM & MDR & 315 & $\mathrm{CC} 1$ \\
\hline SHG-12 & Morocco & 0.125 & 1 & $>8$ & $>8$ & $>8$ & 2 & 64 & $>16$ & $>16$ & $>16$ & $>16$ & $>16$ & OXA-23/NDM & MDR & 2 & $\mathrm{CC} 2$ \\
\hline SHG-16 & Morocco & 0.125 & 1 & $>8$ & $>8$ & $>8$ & 2 & 64 & $>16$ & $>16$ & $>16$ & $>16$ & $>16$ & OXA-23/NDM & MDR & 2 & $\mathrm{CC} 2$ \\
\hline SHG-5 & Morocco & 0.25 & 1 & $>8$ & $>8$ & 2 & 0.125 & 32 & $>16$ & $>16$ & $>16$ & $>16$ & $>16$ & OXA-23 & MDR & 2 & $\mathrm{CC} 2$ \\
\hline SHG-8 & Morocco & 0.25 & 2 & $>8$ & $>8$ & $>8$ & 2 & 64 & $>16$ & $>16$ & $>16$ & $>16$ & $>16$ & OXA-23/NDM & MDR & 2 & CC2 \\
\hline SHG-4 & Morocco & 0.5 & 0.5 & $>8$ & $>8$ & $>8$ & 2 & 64 & $>16$ & $>16$ & $>16$ & $>16$ & $>16$ & OXA-23 & MDR & 2 & $\mathrm{CC} 2$ \\
\hline SHG-7 & Morocco & 0.5 & 0.5 & $>8$ & $>8$ & $>8$ & 2 & 64 & $>16$ & $>16$ & $>16$ & $>16$ & $>16$ & OXA-23 & MDR & 2 & $\mathrm{CC} 2$ \\
\hline SHG-13 & Morocco & 0.5 & 0.5 & 8 & $>8$ & $>8$ & 2 & 64 & $>16$ & $>16$ & $>16$ & $>16$ & $>16$ & OXA-23/NDM & MDR & 632 & $\mathrm{CC} 2$ \\
\hline SHG-14 & Morocco & 1 & 0.25 & $>8$ & $>8$ & $>8$ & 2 & 64 & $>16$ & $>16$ & $>16$ & $>16$ & $>16$ & OXA-23/NDM & MDR & 2 & CC2 \\
\hline SHG-17 & Morocco & 0.5 & 2 & $>8$ & $>8$ & $>8$ & 2 & 64 & $>16$ & $>16$ & $>16$ & $>16$ & $>16$ & OXA-23/NDM & MDR & 164 & CC241 \\
\hline SHG-9 & Morocco & 2 & 0.5 & 0.25 & $>8$ & 0.25 & 0.125 & 16 & $>16$ & $>16$ & $>16$ & $>16$ & $>16$ & OXA-23 & MDR & 164 & CC241 \\
\hline SHG-6 & Morocco & 4 & 0.5 & 0.25 & $>8$ & $>8$ & 1 & 16 & $>16$ & $>16$ & $>16$ & $>16$ & $>16$ & OXA-23 & MDR & 85 & CC85 \\
\hline SHG-25 & Mozambique & 0.03 & 0.5 & 1 & $>8$ & $>8$ & 0.5 & 2 & 8 & 16 & 0.5 & 0.25 & 0.25 & ND & MDR & 23 & CC10 \\
\hline SHG-22 & Mozambique & 0.06 & 0.25 & 0.25 & 0.5 & $>8$ & 0.5 & 4 & 4 & $>16$ & 1 & 0.5 & 0.5 & ND & - & 1435 & CC10 \\
\hline SHG-32 & Mozambique & 0.25 & 0.5 & $>8$ & $>8$ & $>8$ & 2 & 32 & 16 & $>16$ & 4 & 4 & 1 & ND & MDR & 2 & CC2 \\
\hline SHG-18 & Mozambique & 0.5 & 1 & $>8$ & $>8$ & $>8$ & 2 & 32 & $>16$ & $>16$ & 4 & 1 & 0.5 & ND & MDR & 2 & CC2 \\
\hline SHG-29 & Mozambique & 1 & 1 & 2 & $>8$ & $>8$ & 2 & 16 & 2 & $>16$ & 0.5 & 1 & 0.25 & ND & - & 2 & CC2 \\
\hline SHG-24 & Mozambique & 0.06 & 0.5 & 0.25 & 0.125 & 0.25 & 0.125 & 2 & 1 & 2 & 0.125 & 0.125 & 0.25 & ND & - & $\mathrm{New}^{\mathrm{a}}$ & CC216 \\
\hline SHG-27 & Mozambique & 0.5 & 0.25 & 0.5 & $>8$ & $>8$ & 1 & 8 & $>16$ & $>16$ & 1 & 1 & 0.5 & ND & MDR & 164 & CC241 \\
\hline SHG-21 & Mozambique & 1 & 0.25 & 1 & $>8$ & $>8$ & 0.5 & 2 & $>16$ & $>16$ & 1 & 0.5 & 0.5 & ND & MDR & 164 & CC241 \\
\hline SHG-30 & Mozambique & 1 & 0.5 & 0.25 & $>8$ & 0.25 & 0.5 & 8 & $>16$ & $>16$ & 0.5 & 0.5 & 0.25 & ND & - & 164 & CC241 \\
\hline SHG-20 & Mozambique & 0.25 & 0.5 & 0.25 & 0.125 & 0.25 & 0.125 & 2 & 0.5 & 4 & 0.125 & 0.125 & 0.25 & ND & - & 424 & CC33 \\
\hline SHG-19 & Mozambique & 0.125 & 0.5 & 0.25 & $>8$ & $>8$ & 0.25 & 8 & $>16$ & $>16$ & 2 & 2 & 0.5 & ND & MDR & $\mathrm{New}^{\mathrm{a}}$ & CC462 \\
\hline
\end{tabular}


Table 2. Cont.

\begin{tabular}{|c|c|c|c|c|c|c|c|c|c|c|c|c|c|c|c|c|c|}
\hline \multirow{2}{*}{ ID } & \multirow{2}{*}{ Country } & CFDC & CST & MIN & CIP & SXT & TGC & SAM & FEP & CZA & MEM & MEV & IPR & \multirow{2}{*}{ aCarb } & \multirow{2}{*}{ MDR } & \multirow{2}{*}{ ST } & \multirow{2}{*}{$\mathrm{CC}$} \\
\hline & & \multicolumn{12}{|c|}{$\mu \mathrm{g} / \mathrm{mL}$} & & & & \\
\hline SHG-31 & Mozambique & 0.06 & 1 & 0.25 & 0.125 & 0.25 & 0.125 & 2 & 2 & 16 & 0.25 & 0.5 & 0.25 & ND & - & $\mathrm{New}^{\mathrm{a}}$ & CC481 \\
\hline SHG-28 & Mozambique & 0.125 & 0.25 & 0.25 & 0.125 & $>8$ & 0.125 & 2 & 2 & 16 & 0.5 & 0.25 & 0.25 & ND & - & 1359 & CC85 \\
\hline SHG-26 & Mozambique & 0.25 & 0.25 & 0.25 & 0.5 & $>8$ & 0.125 & 2 & 4 & 16 & 0.5 & 4 & 0.25 & ND & - & 1359 & CC85 \\
\hline SHG-34 & Peru & 0.5 & 0.25 & 0.5 & $>8$ & $>8$ & 1 & 16 & $>16$ & $>16$ & $>16$ & $>16$ & $>16$ & OXA-253 & MDR & 7 & $\mathrm{CC} 1$ \\
\hline SHG-33 & Peru & 1 & 1 & 1 & $>8$ & $>8$ & 2 & 64 & $>16$ & $>16$ & $>16$ & $>16$ & $>16$ & OXA-23 & MDR & 1 & CC1 \\
\hline SHG-37 & Peru & 0.125 & 0.5 & 8 & $>8$ & $>8$ & 2 & 32 & $>16$ & $>16$ & $>16$ & $>16$ & $>16$ & OXA-72 & MDR & 2 & $\mathrm{CC} 2$ \\
\hline SHG-36 & Peru & 0.5 & 0.5 & 4 & $>8$ & $>8$ & 1 & 32 & $>16$ & $>16$ & $>16$ & $>16$ & $>16$ & OXA-72 & MDR & 2 & CC2 \\
\hline SHG-40 & Peru & 2 & 0.25 & 8 & $>8$ & $>8$ & 2 & 16 & 16 & $>16$ & $>16$ & $>16$ & $>16$ & OXA-72 & MDR & 108 & $\mathrm{CC} 2$ \\
\hline SHG-39 & Peru & 0.125 & 0.5 & 0.5 & $>8$ & 2 & 0.25 & 16 & $>16$ & $>16$ & $>16$ & $>16$ & $>16$ & OXA-23 & MDR & 3 & CC3 \\
\hline SHG-43 & Peru & 0.5 & 2 & $\leq 0.25$ & 0.25 & 22640.25 & $\leq 0.125$ & $\leq 2$ & 4 & 16 & 0.5 & 0.25 & 0.25 & ND & - & 273 & CC33 \\
\hline SHG-35 & Peru & 0.125 & 0.5 & 0.5 & $>8$ & $>8$ & 2 & 2 & 8 & 16 & 1 & 1 & 0.25 & ND & MDR & 79 & CC79 \\
\hline SHG-38 & Peru & 0.25 & 0.5 & 8 & $>8$ & $>8$ & 2 & 32 & $>16$ & $>16$ & $>16$ & $>16$ & $>16$ & OXA-72 & MDR & 79 & CC79 \\
\hline SHG-41 & Peru & 1 & 0.5 & 1 & $>8$ & $>8$ & 2 & 16 & $>16$ & $>16$ & $>16$ & $>16$ & $>16$ & OXA-253 & MDR & 79 & CC79 \\
\hline SHG-42 & Peru & 2 & 0.25 & 0.5 & $>8$ & $>8$ & 1 & 64 & $>16$ & $>16$ & $>16$ & $>16$ & $>16$ & OXA-23 & MDR & 79 & CC79 \\
\hline SHG-75 & Spain & 0.125 & 2 & $>8$ & $>8$ & $>8$ & 2 & 64 & $>16$ & $>16$ & $>16$ & $>16$ & $>16$ & OXA-58 & MDR & 2 & CC2 \\
\hline SHG-70 & Spain & 0.125 & 0.25 & 8 & $>8$ & $>8$ & 0.5 & 32 & $>16$ & 16 & $>16$ & $>16$ & $>16$ & OXA-23 & MDR & 2 & CC2 \\
\hline SHG-77 & Spain & 0.125 & 1 & 8 & $>8$ & $>8$ & 1 & 32 & 16 & 16 & $>16$ & $>16$ & $>16$ & OXA-58 & MDR & 2 & $\mathrm{CC} 2$ \\
\hline SHG-88 & Spain & 0.125 & 0.5 & 8 & $>8$ & $>8$ & 1 & 16 & $>16$ & 16 & $>16$ & 16 & $>16$ & OXA-23 & MDR & 2 & $\mathrm{CC} 2$ \\
\hline SHG-89 & Spain & 0.125 & 1 & 8 & $>8$ & $>8$ & 2 & 64 & $>16$ & 8 & $>16$ & $>16$ & $>16$ & OXA-23 & MDR & 2 & $\mathrm{CC} 2$ \\
\hline SHG-83 & Spain & 0.25 & 1 & $>8$ & $>8$ & 8 & 2 & 32 & $>16$ & $>16$ & $>16$ & $>16$ & $>16$ & OXA-23 & MDR & 2 & CC2 \\
\hline SHG-78 & Spain & 0.25 & 0.5 & $>8$ & $>8$ & $>8$ & $>4$ & 32 & $>16$ & $>16$ & $>16$ & $>16$ & $>16$ & OXA-23 & MDR & 2 & CC2 \\
\hline SHG-90 & Spain & 0.25 & 0.5 & 8 & $>8$ & $>8$ & 2 & 16 & $>16$ & $>16$ & $>16$ & $>16$ & $>16$ & OXA-23 & MDR & 2 & CC2 \\
\hline SHG-72 & Spain & 0.25 & 0.25 & 4 & $>8$ & 8 & 2 & 32 & $>16$ & $>16$ & $>16$ & $>16$ & $>16$ & OXA-40 & MDR & 2 & $\mathrm{CC} 2$ \\
\hline SHG-74 & Spain & 0.25 & 8 & 2 & $>8$ & $>8$ & 4 & 64 & $>16$ & $>16$ & $>16$ & $>16$ & $>16$ & OXA-23 & MDR & 2 & CC2 \\
\hline SHG-76 & Spain & 0.25 & 0.5 & 4 & $>8$ & $>8$ & 1 & 16 & $>16$ & 16 & $>16$ & $>16$ & $>16$ & OXA-40 & MDR & 2 & CC2 \\
\hline SHG-80 & Spain & 0.5 & 0.5 & $>8$ & $>8$ & $>8$ & $>4$ & 32 & $>16$ & $>16$ & $>16$ & $>16$ & $>16$ & OXA-23 & MDR & 2 & CC2 \\
\hline
\end{tabular}


Table 2. Cont.

\begin{tabular}{|c|c|c|c|c|c|c|c|c|c|c|c|c|c|c|c|c|c|}
\hline \multirow{2}{*}{ ID } & \multirow{2}{*}{ Country } & CFDC & CST & MIN & CIP & SXT & TGC & SAM & FEP & CZA & MEM & MEV & IPR & \multirow{2}{*}{ aCarb } & \multirow{2}{*}{ MDR } & \multirow{2}{*}{ ST } & \multirow{2}{*}{$\mathrm{CC}$} \\
\hline & & \multicolumn{12}{|c|}{$\mu \mathrm{g} / \mathrm{mL}$} & & & & \\
\hline SHG-67 & Spain & 1 & 2 & $>8$ & $>8$ & $>8$ & $>4$ & 16 & $>16$ & $>16$ & 8 & 16 & $>16$ & OXA-58 & MDR & 2 & CC2 \\
\hline SHG-79 & Spain & 1 & 0.5 & 8 & $>8$ & $>8$ & 4 & 32 & $>16$ & $>16$ & $>16$ & $>16$ & $>16$ & OXA-23 & MDR & 2 & CC2 \\
\hline SHG-73 & Spain & 2 & $>8$ & 4 & $>8$ & $>8$ & 4 & 64 & $>16$ & $>16$ & $>16$ & $>16$ & $>16$ & OXA-23 & MDR & 2 & $\mathrm{CC} 2$ \\
\hline SHG-68 & Spain & 2 & 2 & 4 & $>8$ & $>8$ & $>4$ & 32 & $>16$ & $>16$ & $>16$ & $>16$ & $>16$ & OXA-23 & MDR & 2 & $\mathrm{CC} 2$ \\
\hline SHG-85 & Spain & 2 & 0.25 & $>8$ & $>8$ & $>8$ & 1 & 32 & $>16$ & $>16$ & $>16$ & $>16$ & $>16$ & OXA-23* & MDR & 724 & CC2 \\
\hline SHG-69 & Spain & 4 & 0.5 & 4 & $>8$ & $>8$ & $>4$ & 64 & $>16$ & $>16$ & $>16$ & $>16$ & $>16$ & OXA-40 & MDR & 537 & CC79 \\
\hline SHG-81 & Spain & 0.125 & 0.5 & 0.5 & 1 & 0.25 & 0.5 & 16 & 16 & $>16$ & $>16$ & $>16$ & $>16$ & OXA-40 & - & 374 & CC85 \\
\hline SHG-48 & Croatia & 8 & 0.5 & 8 & 8 & 2 & 2 & 32 & $>16$ & 0.125 & $>16$ & $>16$ & $>16$ & OXA-72 & MDR & 2 & CC2 \\
\hline SHG-91 & Azerbaijan & $>64$ & 0.25 & 0.25 & $>8$ & $>8$ & 0.25 & 64 & $>16$ & $>16$ & $>16$ & $>16$ & $>16$ & OXA-23 & MDR & 19 & $\mathrm{CC} 1$ \\
\hline SHG-92 & Azerbaijan & $>64$ & 0.25 & 0.25 & $>8$ & $>8$ & 0.25 & 32 & $>16$ & $>16$ & $>16$ & $>16$ & $>16$ & OXA-23 & MDR & 1423 & $\mathrm{CC} 1$ \\
\hline SHG-107 & Azerbaijan & 32 & 0.5 & 0.25 & $>8$ & $>8$ & 0.25 & $>64$ & $>16$ & $>16$ & $>16$ & $>16$ & $>16$ & OXA-40 & MDR & 78 & CC1017 \\
\hline SHG-111 & Azerbaijan & 64 & 2 & 1 & $>8$ & $>8$ & 1 & 8 & $>16$ & 16 & $>16$ & $>16$ & $>16$ & OXA-40 & MDR & 1077 & CC1017 \\
\hline SHG-113 & Azerbaijan & $>64$ & 0.25 & 4 & $>8$ & $>8$ & 0.5 & 8 & $>16$ & $>16$ & $>16$ & $>16$ & $>16$ & OXA-40 & MDR & 1077 & CC1017 \\
\hline SHG-98 & Azerbaijan & 64 & 0.5 & 2 & $>8$ & $>8$ & 1 & 64 & $>16$ & $>16$ & 0.5 & 0.5 & 0.5 & OXA-40 & MDR & 2 & CC2 \\
\hline SHG-103 & Azerbaijan & $>64$ & 1 & 8 & $>8$ & $>8$ & 2 & $>64$ & $>16$ & $>16$ & $>16$ & $>16$ & $>16$ & OXA-23 & MDR & 2 & CC2 \\
\hline SHG-93 & Azerbaijan & $>64$ & 8 & 8 & $>8$ & 2 & 2 & 8 & $>16$ & $>16$ & $>16$ & $>16$ & $>16$ & OXA-40 & MDR & 2 & CC2 \\
\hline SHG-102 & Azerbaijan & $>64$ & 8.00 & 0.5 & $>8$ & $>8$ & 0.5 & 32 & $>16$ & $>16$ & $>16$ & $>16$ & $>16$ & OXA-40 & MDR & 2 & CC2 \\
\hline SHG-95 & Azerbaijan & $>64$ & 0.5 & 1 & $>8$ & $>8$ & 1 & $>64$ & $>16$ & $>16$ & $>16$ & 16 & $>16$ & OXA-23* & MDR & 2 & CC2 \\
\hline SHG-101 & Azerbaijan & $>64$ & 0.25 & 0.25 & $>8$ & 8 & 0.5 & 8 & $>16$ & $>16$ & 0.5 & 1 & 0.5 & $\mathrm{ND}^{*}$ & MDR & 2 & CC2 \\
\hline SHG-49 & Croatia & 64 & 0.5 & 8 & 8 & 2 & 1 & 64 & $>16$ & $>16$ & $>16$ & $>16$ & $>16$ & OXA-72 & MDR & 2 & CC2 \\
\hline SHG-59 & Greece & 16 & 8 & 2 & $>8$ & $>8$ & 4 & 64 & $>16$ & $>16$ & $>16$ & $>16$ & $>16$ & OXA-23 & MDR & 1 & $\mathrm{CC} 1$ \\
\hline SHG-50 & Greece & $>64$ & 0.25 & $>8$ & $>8$ & $>8$ & 1 & 64 & $>16$ & $>16$ & $>16$ & $>16$ & $>16$ & OXA-23 & MDR & 2 & $\mathrm{CC} 2$ \\
\hline SHG-51 & Greece & $>64$ & 2 & $>8$ & $>8$ & $>8$ & 2 & $>64$ & $>16$ & $>16$ & $>16$ & $>16$ & $>16$ & OXA-23 & MDR & 2 & $\mathrm{CC} 2$ \\
\hline SHG-64 & Italy & $>64$ & 1 & 8 & $>8$ & $>8$ & 1 & 16 & $>16$ & 16 & $>16$ & $>16$ & $>16$ & OXA-23 & MDR & 2 & $\mathrm{CC} 2$ \\
\hline SHG-15 & Morocco & 64 & 0.5 & 4 & $>8$ & $>8$ & 4 & $>64$ & $>16$ & $>16$ & $>16$ & $>16$ & $>16$ & OXA-23/NDM & MDR & 2 & CC2 \\
\hline SHG-3 & Morocco & 16 & 0.5 & 0.25 & $>8$ & $>8$ & 0.25 & 64 & $>16$ & $>16$ & $>16$ & $>16$ & $>16$ & OXA-23 & MDR & 85 & CC85 \\
\hline
\end{tabular}


Table 2. Cont.

\begin{tabular}{|c|c|c|c|c|c|c|c|c|c|c|c|c|c|c|c|c|c|}
\hline \multirow{2}{*}{ ID } & \multirow{2}{*}{ Country } & CFDC & CST & MIN & CIP & SXT & TGC & SAM & FEP & CZA & MEM & MEV & IPR & \multirow{2}{*}{ aCarb } & \multirow{2}{*}{ MDR } & \multirow{2}{*}{ ST } & \multirow{2}{*}{$\mathrm{CC}$} \\
\hline & & \multicolumn{12}{|c|}{$\mu \mathrm{g} / \mathrm{mL}$} & & & & \\
\hline SHG-87 & Spain & $>64$ & 0.5 & $>8$ & $>8$ & $>8$ & 2 & 32 & $>16$ & $>16$ & $>16$ & $>16$ & $>16$ & OXA-23 & MDR & 2 & CC2 \\
\hline SHG-86 & Spain & $>64$ & 0.5 & 1 & $>8$ & $>8$ & 2 & 32 & $>16$ & $>16$ & $>16$ & $>16$ & $>16$ & OXA-23 * & MDR & 2 & CC2 \\
\hline SHG-84 & Spain & $>64$ & 0.25 & 1 & $>8$ & $>8$ & 4 & 64 & $>16$ & $>16$ & $>16$ & $>16$ & $>16$ & OXA-23 * & MDR & 2 & CC2 \\
\hline SHG-71 & Spain & $>64$ & 0.5 & 1 & $>8$ & $>8$ & 4 & 32 & $>16$ & $>16$ & $>16$ & $>16$ & $>16$ & OXA-40* & MDR & 32 & CC85 \\
\hline ATCC19606 & USA & $\leq 0.06$ & 1 & $\leq 0.25$ & $\leq 0.125$ & $>8$ & $\leq 0.125$ & $\leq 2$ & 2 & 8 & 0.5 & 0.5 & 0.25 & ND & - & 52 & - \\
\hline ATCC27853 b & USA & 0.5 & 1 & $>8$ & 0.25 & $>8$ & $>4$ & $>64$ & 1 & 2 & 1 & 0.5 & 0.5 & ND & - & NA & - \\
\hline ATCC25922 ${ }^{c}$ & USA & 0.25 & 0.25 & 1 & $\leq 0.125$ & 0.25 & $\leq 0.125$ & $\leq 2$ & $\leq 0.125$ & $\leq 0.125$ & $\leq 0.06$ & $\leq 0.06$ & 0.25 & ND & - & NA & - \\
\hline
\end{tabular}

ID, strain identification number; Country, country of origin; CFDC, cefiderocol; CST, colistin; MIN, minocycline; CIP, ciprofloxacin; SXT, trimethoprim/sulfamethoxazole; TGC tigecycline; SAM, ampicillin/sulbactam; FEP, cefepime; CZA, ceftazidime/avibactam; MEM, meropenem; MEV, meropenem/vaborbactam; IPR, imipenem/relebactam; aCarb, acquired carbapenemase; MDR, multidrug-resistant; ST, sequence type; CC, clonal complex. ND, not detected. NA, not applicable. ${ }^{\text {a }}$ These isolates presented novel allelic combinations and will be assigned a novel ST number. ${ }^{\mathrm{b}}$ Pseudomonas aeruginosa. ${ }^{\mathrm{c}}$ Escherichia coli. * Positive for PCR screening of bla PER. 


\subsection{Activity of Cefiderocol against Carbapenem-Resistant A. baumannii}

Of the 113 isolates tested, $89(78.76 \%)$ were resistant to meropenem (MIC $\geq 8 \mu \mathrm{g} / \mathrm{mL})$ and such resistance was associated with the carriage of either an OXA-type carbapenemase (79/89) or an OXA-type plus and NDM-type carbapenemase (9/89) in all isolates but one, which was negative for all carbapenemase-encoding genes tested and presented a carbapenem MIC of $8 \mu \mathrm{g} / \mathrm{mL}$, right at the resistance breakpoint (Table 2). All the remaining meropenem non-resistant isolates were also negative for the presence of carbapenemaseencoding genes except for a single isolate carrying $b l a_{\mathrm{OXA}-24}$ that showed a carbapenem MIC of $0.5 \mu \mathrm{g} / \mathrm{mL}$, thus suggesting very low expression of this carbapenemase. In addition, all carbapenem-resistant $A$. baumannii isolates but one showed MIC values of meropenem/vaborbactam and imipenem/relebactam of $\geq 16 \mu \mathrm{g} / \mathrm{mL}$, suggesting little to no activity of these $\beta$-lactam $/ \beta$-lactamase inhibitors. Vaborbactam and relebactam are novel $\beta$-lactamase inhibitors active against class $A$ and class $C \beta$-lactamases but are not able to inhibit class B or D carbapenemases, which are common in carbapenem-resistant A. baumannii isolates [13]. The only carbapenem-resistant isolate showing reduced MICs ofmeropenem/vaborbactam and imipenem/relebactam (MIC of $2 \mu \mathrm{g} / \mathrm{mL}$ and $1 \mu \mathrm{g} / \mathrm{mL}$, respectively) was negative for bla genes encoding class B or class D carbapenemases, thus suggesting the presence of additional resistance mechanisms such as expression of a class A carbapenemase that would be inhibited by the $\beta$-lactamase inhibitor in the combination (bla $a_{\mathrm{PER}}$ or $b l a_{\mathrm{VEB}}$ were not detected in this isolate either).

On the other hand, 75.28\% (67/89) of carbapenem-resistant isolates were susceptible to cefiderocol, clearly showing superior activity of cefiderocol compared to the novel $\beta$-lactam/ $\beta$-lactamase inhibitors. Interestingly, all cefiderocol non-susceptible isolates $(24 / 113)$ were also resistant to meropenem except for two isolates, one carrying an OXA-40 oxacillinase and the other carrying a PER-1 class A $\beta$-lactamase (Table 2). Carriage of an OXA-23 oxacillinase $(13 / 24,54.16 \%)$ was the main mechanism associated with carbapenemresistance among these isolates, closely followed by carriage of OXA-40 (8/24, 33.33\%) and OXA-72 (2/24, 8.33\%). These findings were in line with previous studies in which the highest MICs to cefiderocol (MIC $\geq 8 \mu \mathrm{g} / \mathrm{mL}$ ) were related toisolates harbouring OXA-23 and/or OXA-40, although such results were likely biased by the fact that these two mechanisms are by far the most common mechanisms of resistance among carbapenem-resistant clinical isolates of A. baumannii, and it is important to highlight that the only carbapenemsusceptible isolate lacking an oxacillinase within this group presented cefiderocol MIC values of $>64 \mu \mathrm{g} / \mathrm{mL}$ [7,14-16]. In line with the above, the main carbapenem-resistant mechanism detected among cefiderocol-susceptibleisolates in our study was also OXA-23, present in $52.8 \%$ of the isolates (47/89), followed again by OXA-40 (9/89; 10.11\%), OXA-72 $(5 / 89 ; 5.51 \%)$ as well as a few isolates harbouring OXA-58 and OXA-253 (3.37\% and $2.24 \%$, respectively).

Interestingly, Kohira et al. recently suggested that NDM might have a role in cefiderocol non-susceptibility among Enterobacterales and PER might also be involved in a slight MIC increase of cefiderocol in A. baumannii, although the expression of PER enzymes alone did not seem to result in cefiderocol MIC values of $>8 \mu \mathrm{g} / \mathrm{mL}$ and PER-positive A. baumannii isolates in such a study were closely clonally related [17]. Notably, only one out of nine isolates harbouring OXA-23/NDM in our study developed resistance to cefiderocol and only 5 out of 24 cefiderocol non-susceptible isolates were positive for bla $a_{\mathrm{PER}}$, all but one associated to sequence type 2 (ST2). We also identified a sixth PER-positive meropenem-resistant $A$. baumannii isolate in our collection that, nevertheless, showed a cefiderocol MIC of $2 \mu \mathrm{g} / \mathrm{mL}$ (Table 2). The distribution of OXA, NDM and PER enzymes between resistant and susceptible isolates to cefiderocol in our collection, therefore, seemed to suggest that there is no link between cefiderocol-resistance and the carriage of a particular $\beta$-lactam-resistance gene. 


\subsection{Geographical and Epidemiological Distribution of Cefiderocol-Resistant Isolates}

A. baumannii isolates selected in the present study originated from nine different countries from four different continents in an attempt to provide some degree of epidemiological diversity. In addition, isolates could be grouped in at least 29 different STs from 13 clonal complexes, including isolates from the widespread international clonal complexes CC1, CC2, and CC3 (Table 2) [18]. Interestingly, however, while cefiderocol-susceptible isolates were recovered from all nine participating countries, cefiderocol non-susceptible isolates were only recovered from six out of the nine countries included, and there was a clear predominance towards isolates recovered from Azerbaijan (12/24,50\%) as well as towards isolates belonging to CC2 $(15 / 24,62,5 \%)$, as shown in Figure 1 and Table 2 . According to PFGE data, however, there was little clonal homogeneity among the cefiderocol-resistant isolates from Azerbaijan (data not shown) and, in general, there was a strong bias in our collection towards isolates belonging to CC2 as well $(62 / 113,54.8 \%)$, which is just a mere reflection of the worldwide spread of such international clone [18]. For instance, we did not detect cefiderocol-resistance among isolates recovered from Peru, Argentina or Mozambique, despite some of them also belonging to CC2.

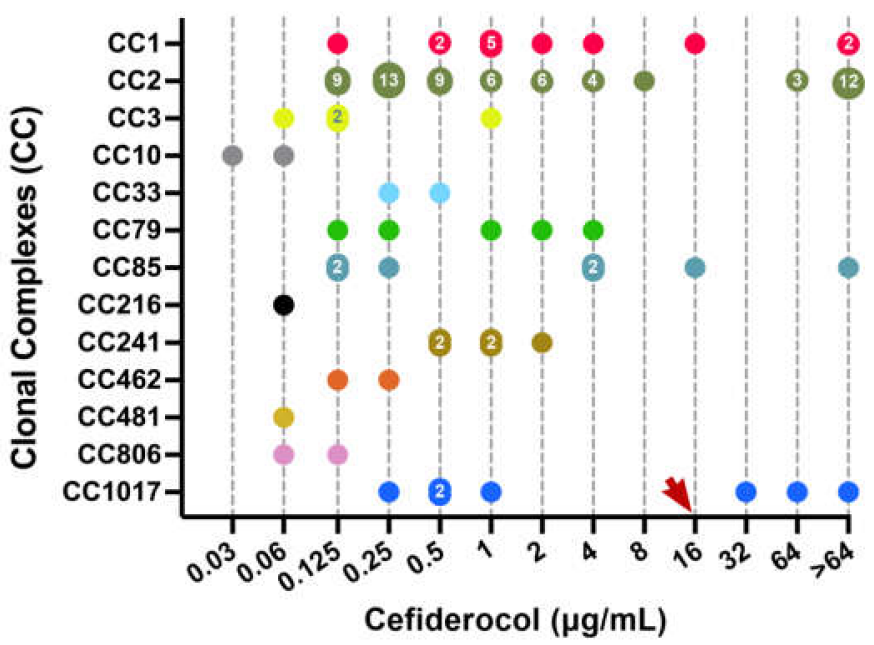

Figure 1. Distribution of all isolates according to their cefiderocol MIC and classification into main clonal complexes (CC). Numbers within data points indicate the number of isolates with the same MIC value. The red arrow shows the CLSI clinical breakpoint for cefiderocol $(\geq 16 \mu \mathrm{g} / \mathrm{mL})$.

In view of the above, it is difficult to associate cefiderocol-resistance with a particular clonal lineage, although there seemed to be a certain preference in our study towards isolates from CC2. In this regard, Malik et al. also suggested an association between A. baumannii ST2 isolates and cefiderocol-resistance, linked to the reduced expression of the bacterial siderophore receptor PirA [19]. While the expression of siderophore receptors in different clonal groups has not yet been investigated in our group, it is likely that the emergence of cefiderocol resistance is strain specific and that the putative association with isolates from CC2 arises from the fact that mutations affecting such expression are most likely to be identified first among isolates from predominant clonal lineages, that are far more abundant in clinical settings. Nevertheless, resistance mechanisms in A. baumannii have typically been shown to be multifactorial, often involving modifications on membrane permeability and moiety but also biofilm formation, so additional epidemiological and molecular studies are clearly needed to elucidate the mechanisms behind cefiderocol resistance $[20,21]$. 


\section{Materials and Methods}

\subsection{Antimicrobial Agents}

Antibiotics tested in this study were previously prepared in frozen 96-well microtiter plates containing twofold serial dilutions of: cefepime, cefiderocol, ceftazidime-avibactam, imipenem-relebactam, ampicillin-sulbactam, meropenem, meropenem-vaborbactam, ciprofloxacin, minocycline, tigecycline, trimethoprim-sulfamethoxazole and colistin. The same plates and conditions were used for all strains. In all cases except for cefiderocol, the broth microdilution procedure was performed in cation-adjusted Mueller-Hinton broth (CAMHB). For cefiderocol iron-depleted, CAMHB was used and prepared following CLSI-approved methodology [22].

\subsection{Bacterial Isolates}

A collection of 113 epidemiologically diverse clinical isolates of $A$. baumannii recovered from Argentina $(n=4)$, Azerbaijan $(n=23)$, Croatia $(n=3)$, Greece $(n=10)$, Italy $(n=7)$, Morocco $(n=17)$, Mozambique $(n=14)$, Peru $(n=11)$ and Spain $(n=24)$ were selected for this study. For the interpretation of the results, available CLSI breakpoints were applied to determine categories (susceptible, intermediate and resistant) [22]. The A. baumannii ATCC 19606, P. aeruginosa ATCC 27,853 and E. coli ATCC 25,922 were used as quality control strains.

\subsection{Minimum Inhibitory Concentration (MIC) Determinations}

Antimicrobial susceptibility was performed according to the standard CLSI methodology [22]. After inoculation plates were placed in aerobic conditions and readings were taken after 16-20 h of incubation. The MIC was determined as the lowest concentration of an individual drug that resulted in no visible growth. $\mathrm{MIC}_{50}$ and $\mathrm{MIC}_{90}$ values were also calculated and indicated the MIC value of each drug able to inhibit the growth of $50 \%$ and $90 \%$ of isolates tested, respectively.

\subsection{Molecular Identification of Carbapenem-Resistance Genes}

The presence of the serine class A carbapenemases ( $b l a_{\mathrm{KPC}}, b l a_{\mathrm{PER}}$ and $\left.b l a_{\mathrm{VEB}}\right)$, class $\mathrm{B}$ MBLs $\left(b l a_{\mathrm{NDM}}, b l a_{\mathrm{IMP}}, b l a_{\mathrm{VIM}}, b l a_{\mathrm{SPM}}\right.$, and $\left.b l a_{\mathrm{SIM}}\right)$ and class D oxacillinases (bla $a_{\mathrm{OXA}-51}$-like, $b l a_{\mathrm{OXA}-23}$-like, $b l a_{\mathrm{OXA}-24}$-like, $b l a_{\mathrm{OXA}-58}$-like, $b l a_{\mathrm{OXA}-143}$-like, and $b l a_{\mathrm{OXA}-235}$-like) was checked by PCR, as previously described $[23,24]$.

\subsection{Multi-Locus Sequence Typing (MLST)}

MLST was performed using the Pasteur scheme for A. baumannii [25]. The allele sequences and STs of selected strains were identified and retrieved from the PubMLST A. baumannii MLST database (http://pubmlst.org/abaumannii/ (last accessed on 17 December 2021). The population structure of STs was assigned to their corresponding clonal complexes (CCs) using the goeBURST software (http:/ / www.phyloviz.net/goeburst/ (last accessed on 17 December 2021)).

\section{Conclusions}

Overall, almost $80 \%$ of $A$. baumannii isolates in our study showed susceptibility to cefiderocol, and, more interestingly, as many as $75 \%$ of meropenem-resistant isolates were also susceptible to the new drug. Despite the fact that most cefiderocol-resistant isolates belong to $\mathrm{CC} 2$, our data seem to suggest that cefiderocol-resistance is strain specific and cannot be related to any particular mechanism of carbapenem-resistance either.

There are already several publications that have investigated the in vitro activity of cefiderocol against clinical isolates of A. baumannii [7,26,27]. Such publications, however, usually fail to consider the clonal relatedness of isolates selected for the study and, given the strong clonal structure of A. baumannii, is it likely that they lack in clonal diversity. In our study we have tried not to focus on including a sheer number of isolates but to provide as much clonal diversity as possible. The susceptibility profiles from our collection of 
epidemiologically diverse isolates harboring different mechanisms of carbapenem-resistance, nevertheless, reinforce previous results that prompted cefiderocol as a valid alternative to treat infections caused by MDR A. baumannii.

Author Contributions: Conceptualization: C.B.-D., C.L., I.R. and J.V.; methodology: Á.R., L.M. and I.R.; validation: C.B.-D. and Á.R.; formal analysis: C.B.-D., Á.R., I.R. and J.V.; investigation: C.B.-D., Á.R., L.M., I.R. and J.V.; data curation: Á.R. and I.R.; writing—original draft preparation: C.B.-D., Á.R., C.L., I.R. and J.V.; writing-review and editing: I.R; visualization: Á.R. and I.R.; supervision: C.B.-D., C.L. and J.V.; project administration: C.B.-D., L.M. and J.V.; funding acquisition: C.B.-D., C.L., I.R. and J.V. All authors have read and agreed to the published version of the manuscript.

Funding: This study was supported by Plan Nacional de I+D+i 2013-2016, Instituto de Salud Carlos III, Subdirección General de Redes y Centros de Investigación Cooperativa, Ministerio de Economía y Competitividad, Spanish Network for Research in Infectious Diseases (REIPI RD16/0016/0010); the 2017 call for Strategic Action on Health (PI17/01932 and PI17/01468), co-financed by European Development Regional Fund "A way to achieve Europe" and the operative program Intelligent Growth 2014-2020; grant 2017 SGR 0809 from the Departament d'Universitats, Recerca i Societat de la Informació, of the Generalitat de Catalunya; and the COMBACTECARE project (Grant Number 115620). I.R. was supported by the Department of Health, Generalitat de Catalunya, grant SLT002/16/00349. We also acknowledge support from the Spanish Ministry of Science, Innovation and Universities through the "Centro de Excelencia Severo Ochoa 2019-2023" Program (CEX2018-000806-S), and support from the Generalitat de Catalunya through the CERCA Program". Additional financial support and provision of custom frozen microdilution plates containing antibiotics were received from Shionogi B.V. as a research grant.

Institutional Review Board Statement: Not applicable.

Informed Consent Statement: Not applicable.

Data Availability Statement: The datasets generated by this study are available in Table 2.

Acknowledgments: We thank the team of curators of the Institute Pasteur MLST and whole genome MLST databases for curating the data and making them publicly available at http:/ / bigsdb.pasteur.fr / (last accessed on 17 December 2021).

Conflicts of Interest: C.L. is an employee of Shionogi and J.V. has received a research grant, and from Shionogi.

\section{References}

1. Roca, I.; Espinal, P.; Vila-Farrés, X.; Vila, J. The Acinetobacter baumannii oxymoron: Commensal hospital dweller turned pan-drug-resistant menace. Front. Microbiol. 2012, 3, 148. [CrossRef] [PubMed]

2. Tacconelli, E.; Carrara, E.; Savoldi, A.; Harbarth, S.; Mendelson, M.; Monnet, D.L.; Pulcini, C.; Kahlmeter, G.; Kluytmans, J.; Carmeli, Y.; et al. Discovery, research, and development of new antibiotics: The WHO priority list of antibiotic-resistant bacteria and tuberculosis. Lancet Infect. Dis. 2018, 18, 318-327. [CrossRef]

3. European Centre for Disease Prevention and Control (eCDC). Surveillance Report. Surveillance of Antimicrobial Resistance in Europe 2018. Available online: https:/ / www.ecdc.europa.eu/en/publications-data/surveillance-antimicrobial-resistanceeurope-2018 (accessed on 12 November 2021).

4. Center for Disease Control and Prevention (U.S.). Antibiotic Resistance Threats in the United States, Department of Health and Human Services, CDC. Available online: https://www.cdc.gov/DrugResistance/Biggest-Threats.html (accessed on 12 November 2021).

5. Isler, B.; Doi, Y.; Bonomo, R.A.; Paterson, D.L. New treatment options against carbapenem-resistant Acinetobacter baumannii infections. Antimicrob. Agents Chemother. 2019, 63, 63. [CrossRef] [PubMed]

6. Vila, J.; Moreno-Morales, J.; Ballesté-Delpierre, C. Current landscape in the discovery of novel antibacterial agents. Clin. Microbiol. Infect. 2020, 26, 596-603. [CrossRef] [PubMed]

7. Karlowsky, J.A.; Hackel, M.A.; Tsuji, M.; Yamano, Y.; Echols, R.; Sahm, D.F. In vitro activity of cefiderocol, a siderophore cephalosporin, against gram-negative bacilli isolated by clinical laboratories in North America and Europe in 2015-2016: SIDEROWT-2015. Int. J. Antimicrob. Agents 2019, 53, 456-466. [CrossRef]

8. El-Lababidi, R.M.; Rizk, J.G. Cefiderocol: A siderophore cephalosporin. Ann. Pharmacother. 2020, 54, 1215-1231. [CrossRef] 
9. U.S. Food and Drug Administration. FDA Approves New Antibacterial Drug to Treat Complicated Urinary Tract Infections as Part of Ongoing Efforts to Address Antimicrobial Resistance. Available online: https://www.fda.gov/news-events/ press-announcements / fda-approves-new-antibacterial-drug-treat-complicated-urinary-tract-infections-part-ongoing-efforts (accessed on 12 November 2021).

10. European Medicines Agency (EMA). Fetcroja (Cefiderocol). Available online: https://www.ema.europa.eu/en/medicines/ human/EPAR/fetcroja (accessed on 12 November 2021).

11. Magiorakos, A.-P.; Srinivasan, A.; Carey, R.; Carmeli, Y.; Falagas, M.; Giske, C.; Harbarth, S.; Hindler, J.; Kahlmeter, G.; OlssonLiljequist, B.; et al. Multidrug-resistant, extensively drug-resistant and pandrug-resistant bacteria: An international expert proposal for interim standard definitions for acquired resistance. Clin. Microbiol. Infect. 2012, 18, 268-281. [CrossRef]

12. Lashinsky, J.N.; Henig, O.; Pogue, J.; Kaye, K.S. Minocycline for the Treatment of Multidrug and Extensively Drug-Resistant A. baumannii: A Review. Infect. Dis. Ther. 2017, 6, 199-211. [CrossRef]

13. Novelli, A.; Del Giacomo, P.; Rossolini, G.M.; Tumbarello, M. Meropenem/vaborbactam: A next generation $\beta$-lactam $\beta$-lactamase inhibitor combination. Expert Rev. Anti-Infect. Ther. 2020, 18, 643-655. [CrossRef]

14. Dobias, J.; Dénervaud-Tendon, V.; Poirel, L.; Nordmann, P. Activity of the novel siderophore cephalosporin cefiderocol against multidrug-resistant Gram-negative pathogens. Eur. J. Clin. Microbiol. 2017, 36, 2319-2327. [CrossRef]

15. Ito, A.; Kohira, N.; Bouchillon, S.K.; West, J.; Rittenhouse, S.; Sader, H.; Rhomberg, P.; Jones, R.N.; Yoshizawa, H.; Nakamura, R.; et al. In vitro antimicrobial activity of S-649266, a catechol-substituted siderophore cephalosporin, when tested against non-fermenting Gram-negative bacteria. J. Antimicrob. Chemother. 2016, 71, 670-677. [CrossRef] [PubMed]

16. Boral, B.; The Acinetobacter Study Group; Unaldi, Ö.; Ergin, A.; Durmaz, R.; Eser, Ö.K. A prospective multicenter study on the evaluation of antimicrobial resistance and molecular epidemiology of multidrug-resistant Acinetobacter baumannii infections in intensive care units with clinical and environmental features. Ann. Clin. Microbiol. Antimicrob. 2019, 18, 1-9. [CrossRef] [PubMed]

17. Kohira, N.; Hackel, M.A.; Ishioka, Y.; Kuroiwa, M.; Sahm, D.F.; Sato, T.; Maki, H.; Yamano, Y. Reduced susceptibility mechanism to cefiderocol, a siderophore cephalosporin, among clinical isolates from a global surveillance programme (SIDERO-WT-2014). $J$. Glob. Antimicrob. Resist. 2020, 22, 738-741. [CrossRef] [PubMed]

18. Karah, N.; Sundsfjord, A.; Towner, K.; Samuelsen, Ø. Insights into the global molecular epidemiology of carbapenem nonsusceptible clones of Acinetobacter baumannii. Drug Resist. Updates 2012, 15, 237-247. [CrossRef] [PubMed]

19. Malik, S.; Kaminski, M.; Landman, D.; Quale, J. Cefiderocol resistance in Acinetobacter baumannii: Roles of $\beta$-lactamases, siderophore receptors, and penicillin binding protein 3. Antimicrob. Agents Chemother. 2020, 64, e01221-20. [CrossRef] [PubMed]

20. Trebosc, V.; Gartenmann, S.; Tötzl, M.; Lucchini, V.; Schellhorn, B.; Pieren, M.; Lociuro, S.; Gitzinger, M.; Tigges, M.; Bumann, D.; et al. Dissecting colistin resistance mechanisms in extensively drug-resistant Acinetobacter baumannii clinical isolates. $m B i o$ 2019, 10, e01083-19. [CrossRef] [PubMed]

21. Donadu, M.G.; Mazzarello, V.; Cappuccinelli, P.; Zanetti, S.; Madléna, M.; Nagy, Á.L.; Stájer, A.; Burián, K.; Gajdács, M. Relationship between the biofilm-forming capacity and antimicrobial resistance in clinical Acinetobacter baumannii isolates: Results from a laboratory-based in vitro study. Microorganisms 2021, 9, 2384. [CrossRef]

22. Clinical and Laboratory Standards Institute (CLSI). Performance Standards for Antimicrobial Susceptibility Testing. Thirty Informational Supplement, M100-S30; Clinical and Laboratory Standards Institute: Wayne, PA, USA, 2020.

23. Blitchtein, S.L.; Roca, I.; Rebata, S.P.; Vicente-Taboada, W.; Velásquez-Pomar, J.; Muñoz, L.; Moreno-Morales, J.; Pons, M.J.; Mendoza, J.D.V.; Vila, J. Emergence and spread of carbapenem-resistant Acinetobacter baumannii international clones II and III in Lima, Peru. Emerg. Microbes Infect. 2018, 7, 1-9. [CrossRef]

24. Bogaerts, P.; de Castro, R.R.; de Mendonca, R.; Huang, T.-D.; Denis, O.; Glupczynski, Y. Validation of carbapenemase and extended-spectrum -lactamase multiplex endpoint PCR assays according to ISO 15189. J. Antimicrob. Chemother. 2013, 68, 1576-1582. [CrossRef]

25. Diancourt, L.; Passet, V.; Nemec, A.; Dijkshoorn, L.; Brisse, S. The population structure of Acinetobacter baumannii: Expanding multiresistant clones from an ancestral susceptible genetic pool. PLoS ONE 2010, 5, e10034. [CrossRef]

26. Hackel, M.A.; Tsuji, M.; Yamano, Y.; Echols, R.; Karlowsky, J.A.; Sahm, D.F. In vitro activity of the siderophore cephalosporin, cefiderocol, against carbapenem-nonsusceptible and multidrug-resistant isolates of gram-negative bacilli collected worldwide in 2014 to 2016. Antimicrob. Agents Chemother. 2018, 62, e01968-17. [CrossRef] [PubMed]

27. Golden, A.R.; Adam, H.J.; Baxter, M.; Walkty, A.; Lagacé-Wiens, P.; Karlowsky, J.A.; Zhanel, G.G. In vitro activity of cefiderocol, a novel siderophore cephalosporin, against gram-negative bacilli isolated from patients in Canadian intensive care units. Diagn. Microbiol. Infect. Dis. 2020, 97, 115012. [CrossRef] [PubMed] 